Article

\title{
Parsec-Scale Structure and Kinematics of Faint TeV HBLs
}

\author{
B. Glenn Piner ${ }^{1}$ and Philip G. Edwards ${ }^{2, *}$ \\ 1 Department of Physics and Astronomy, Whittier College, Whittier, CA 90608, USA; gpiner@whittier.edu \\ 2 CSIRO Astronomy and Space Science, Epping 2121, Australia \\ * Correspondence: philip.edwards@csiro.au; Tel.: +61-2-9372-4717
}

Academic Editors: Jose L. Gómez, Alan P. Marscher and Svetlana G. Jorstad

Received: 6 September 2016; Accepted: 30 September 2016; Published: 11 October 2016

\begin{abstract}
We present new multi-epoch Very Long Baseline Array (VLBA) observations of a set of TeV blazars drawn from our VLBA program to monitor all TeV-detected high-frequency peaked BL Lac objects (HBLs) at parsec scales. Most of these sources are faint in the radio, so they have not been well observed with VLBI by other surveys. Our previous measurements of apparent jet speeds in TeV HBLs showed apparent jet speeds that were subluminal or barely superluminal, suggesting jets with velocity structures at the parsec-scale. Here we present apparent jet speed measurements for eight new TeV HBLs, which for the first time show a superluminal tail to the apparent speed distribution for the TeV HBLs.
\end{abstract}

Keywords: TeV blazars; VLBI; active galactic nuclei

\section{Introduction}

At $\mathrm{TeV}$ energies $\left(10^{12} \mathrm{eV}\right)$, three orders of magnitude higher than those studied by satellite-based detectors, gamma-ray astronomy is conducted with ground based telescopes such as H.E.S.S., VERITAS and MAGIC. Over $175 \mathrm{TeV}$ gamma-ray sources have now been catalogued (http://tevcat.uchicago.edu/), with over one third of these being extragalactic objects. The majority of these (46 of 69) are classified as HBL (High-frequency-peaked BL Lac) objects, for which the synchrotron peak of the Spectral Energy Distribution (SED) lies at frequencies above $10^{16.5} \mathrm{~Hz}$.

Many of the well-studied HBL TeV sources have shown dramatic variability in their gamma-ray emission [1,2]. The most rapid variations suggest extremely small emitting volumes and/or time compression by large relativistic Doppler factors of up to $\sim 100$ and challenge our understanding of relativistic jets [2-4].

The only way to directly obtain information on the parsec-scale structure of these blazar jets is by imaging the radio sources using the technique of Very Long Baseline Interferometry (VLBI). However, many HBLs are fainter at radio wavelengths (typically tens of milli-janskys-see Table 1) than the more powerful quasars and BL Lac objects, and are not included in VLBI monitoring programs such as MOJAVE [5] and TANAMI [6]. Properties that can be measured from VLBI images-the apparent jet speed, radio core brightness temperature, core dominance, and jet-to-counter-jet brightness ratio-provide information on fundamental properties of the jet, such as the bulk Lorentz factor and viewing angle.

Yet, despite the high Doppler factors inferred from $\mathrm{TeV}$ observations, our previous VLBA observations have established that $\mathrm{TeV}$ sources have only modest brightness temperatures, and jet component motions that are sub-luminal or only slightly superluminal [7-15]. This has been called the "doppler crisis" [16] or "bulk Lorentz factor crisis" [17]. 
Table 1. Current status of our Very Long Baseline Array (VLBA) monitoring program. TeV source names are those used by TeVCat, redshifts are those given in [15], where an asterisk denotes a tentative value or limit. The NRAO VLA Sky Survey (NVSS) flux density is measured at $1.4 \mathrm{GHz}$. The number of epochs refers to the number of VLBA images made in our monitoring program. References are to papers presenting these image.

\begin{tabular}{|c|c|c|c|c|c|}
\hline TeV Source Name & Redshift & NVSS Counterpart & $\begin{array}{l}\text { NVSS Flux } \\
\text { Density (mJy) }\end{array}$ & $\begin{array}{l}\text { Number } \\
\text { of Epochs }\end{array}$ & References \\
\hline SHBL J001355.9 - 185406 & 0.094 & NVSS J001356 - 185406 & 29.2 & 4 & [15] \\
\hline KUV 00311 - 1938 & $0.506^{*}$ & NVSS J003334 - 192133 & 18.5 & 4 & [15] \\
\hline 1ES $0033+595$ & $0.240 *$ & NVSS J003552 + 595005 & 147.3 & 5 & [15] \\
\hline RGB J0136 + 391 & $0.400 *$ & NVSS J013632 + 390559 & 60.0 & 4 & [15] \\
\hline RGB J0152 + 017 & 0.080 & NVSS J015239 + 014717 & 61.4 & 5 & [15] \\
\hline $1 \mathrm{ES} 0229+200$ & 0.140 & NVSS J023248 + 201716 & 82.4 & 5 & {$[15,18]$} \\
\hline PKS $0301-243$ & 0.266 & NVSS J030326 - 240710 & 700.2 & 2 & - \\
\hline IC 310 & 0.019 & NVSS J031642 + 411928 & 168.1 & 2 & - \\
\hline RBS 0413 & 0.190 & NVSS J031951 + 184536 & 20.9 & 5 & {$[15,18]$} \\
\hline $1 \mathrm{ES} 0347-121$ & 0.188 & NVSS J034922 - 115914 & 23.9 & 5 & {$[15,18]$} \\
\hline $1 \mathrm{ES} 0414+009$ & 0.287 & NVSS J041652 + 010526 & 119.6 & 5 & {$[15,18]$} \\
\hline $1 \mathrm{ES} 0502+675$ & 0.314 & NVSS J050755 + 673724 & 25.4 & 5 & {$[15,18]$} \\
\hline PKS 0548 - 322 & 0.069 & NVSS J055040 - 321620 & 343.7 & 5 & {$[15,18]$} \\
\hline RX J0648.7 + 1516 & 0.179 & NVSS J064847 + 151625 & 64.2 & 4 & [15] \\
\hline $1 \mathrm{ES} 0647+250$ & 0.450 & NVSS J065046 + 250259 & 96.2 & 5 & [15] \\
\hline RGB J0710 + 591 & 0.125 & NVSS J071030 + 590817 & 158.4 & 5 & {$[15,18]$} \\
\hline $1 \mathrm{ES} 0806+524$ & 0.138 & NVSS J080949 + 521858 & 182.4 & 3 & [18] \\
\hline RBS 0723 & 0.198 & NVSS J084712 + 113350 & 32.8 & 1 & - \\
\hline 1RXS J101015.9 - 311909 & 0.143 & NVSS J101015 - 311906 & 73.5 & 4 & [15] \\
\hline 1ES $1011+496$ & 0.212 & NVSS J101504 + 492601 & 377.7 & 3 & [18] \\
\hline 1ES $1101-232$ & 0.186 & NVSS J110337 - 232924 & 120.3 & 5 & [14] \\
\hline Markarian 421 & 0.031 & NVSS J110427 + 381232 & 767.4 & 17 & {$[7,11,19]$} \\
\hline Markarian 180 & 0.045 & NVSS J113626 + 700925 & 327.1 & 7 & [14] \\
\hline RX J1136.5 + 6737 & 0.134 & NVSS J113629 + 673706 & 45.3 & 1 & - \\
\hline $1 \mathrm{ES} 1215+303$ & 0.130 & NVSS J121752 + 300700 & 571.6 & 2 & - \\
\hline $1 \mathrm{ES} 1218+304$ & 0.184 & NVSS J122121 + 301036 & 71.0 & 5 & [14] \\
\hline MS $1221.8+2452$ & 0.218 & NVSS J122424 + 243623 & 24.5 & 4 & {$[15]$} \\
\hline H $1426+428$ & 0.129 & NVSS J142832 + 424022 & 58.0 & 5 & {$[12,19]$} \\
\hline $1 \mathrm{ES} 1440+122$ & 0.163 & NVSS J144248 + 120040 & 68.8 & 4 & {$[15]$} \\
\hline PG $1553+113$ & $0.500 *$ & NVSS J155543 + 111124 & 312.0 & 7 & {$[14]$} \\
\hline Markarian 501 & 0.034 & NVSS J165352 + 394536 & 1558.0 & 9 & {$[8,13,19]$} \\
\hline H $1722+119$ & $0.170 *$ & NVSS J172504 + 115215 & 120.4 & 4 & [15] \\
\hline $1 \mathrm{ES} 1727+502$ & 0.055 & NVSS J172818 + 501311 & 200.7 & 2 & - \\
\hline $1 \mathrm{ES} 1741+196$ & 0.083 & NVSS J174357 + 193508 & 301.2 & 4 & [15] \\
\hline $1 \mathrm{ES} 1959+650$ & 0.047 & NVSS J195959 + 650854 & 249.6 & 13 & {$[10,11,19]$} \\
\hline PKS $2155-304$ & 0.116 & NVSS J215852 - 301330 & 489.3 & 7 & {$[10,11,19]$} \\
\hline B3 $2247+381$ & 0.119 & NVSS J225005 + 382437 & 103.4 & 4 & [15] \\
\hline $1 \mathrm{ES} 2344+514$ & 0.044 & NVSS J234705 + 514217 & 250.4 & 8 & {$[10,19]$} \\
\hline Н $2356-309$ & 0.165 & NVSS J235907 - 303740 & 62.1 & 5 & [14] \\
\hline
\end{tabular}

Of the 46 HBLs detected at TeV energies:

- 11 have jet kinematics published previously by us. (Some of these are also in MOJAVE.)

- 7 have, or soon will have, speeds determined by the MOJAVE program [5].

- 20 are included in the current phase of our program, with first epoch VLBA results for all 20 published [15]. We present here the kinematic results for the first portion of these sources.

- 4 are too far south to be studied with the VLBA, with several of these are part of the TANAMI monitoring program [6].

- 4 are recent detections which are yet to be monitored with VLBI.

The $39 \mathrm{TeV}$ sources for which we have at least one VLBA image are listed in Table 1, together with their redshift, association in the NRAO VLA Sky Survey (NVSS) catalog [20], 1.4 GHz flux 
density, and details of our VLBA observations. Images and data available at the project website: www2.whittier.edu/facultypages/gpiner/research/archive/archive.html.

$\mathrm{TeV}$ photons are attenuated by the infrared background [21] and, as is apparent in Table 1, the majority of extragalactic $\mathrm{TeV}$ sources are at relatively low redshift $(z<0.2)$. Conversely, studies of $\mathrm{TeV}$ gamma-ray spectra have offered a means of constraining the infrared background [21,22].

\section{Results}

Images of four of the sources currently being monitored are shown in Figure 1. All show parsec-scale morphologies typical of this class: a compact core (which hosts the supermassive black hole powering the source), and a weaker, one-sided jet that transitions to a decollimated structure with larger opening angle at a few tens of milli-arcseconds from the core (see image of 0229+200). Multi-epoch studies of these jets over the course of several years allow the apparent speeds of the jet components to be determined.

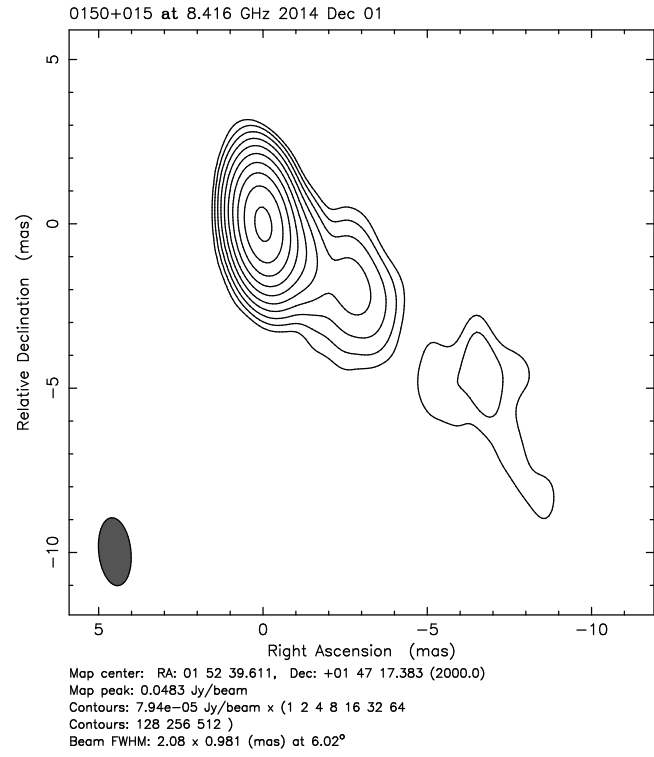

(a)

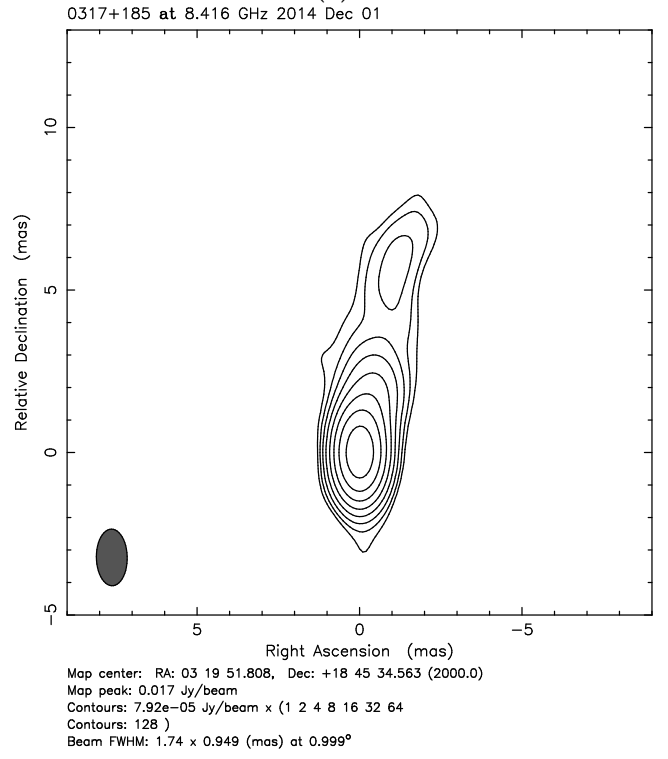

(c)

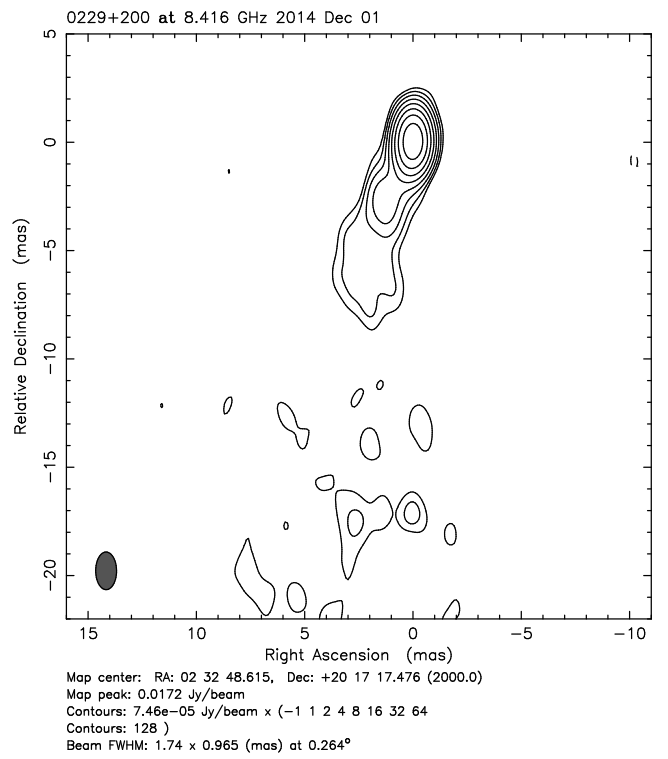

(b)

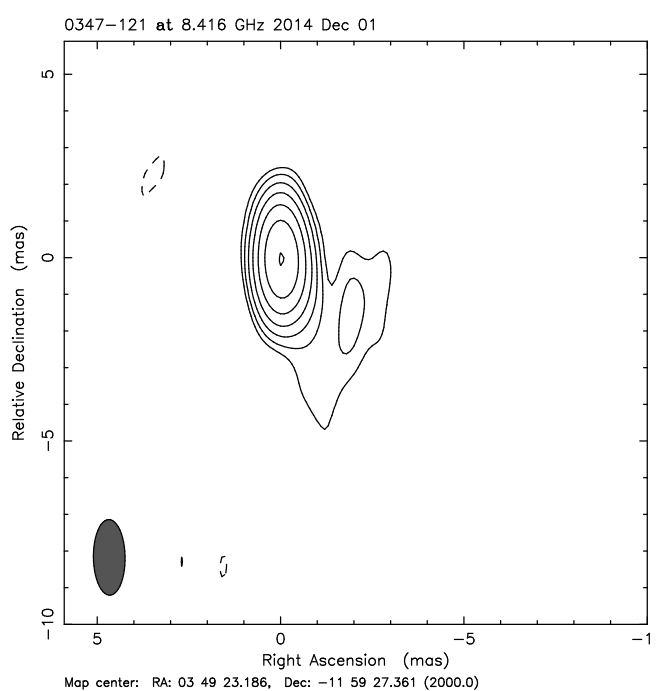

Map center: RA: $034923.186, \quad$ Dec: $-115927.361(2000.0)$
Map peak: 0.00571 Jy/beom Contours: $8.78 \mathrm{e}-05 \mathrm{Jy} /$ beam $\times(-1) 248163264)$

(d)

Figure 1. VLBA images at $8 \mathrm{GHz}$ of four Tev blazars in out VLBA monitoring program: (a) RGB J0152+017; (b) 1ES 0229+200; (c) RBS 0413 (0317+185); (d) 1ES 0347-121. 
Our previous VLBA studies indicated the absence of rapidly moving features in the jets of $\mathrm{TeV}$ HBLs; jet components were either nearly stationary or slowly moving $(<\sim 1 c)$ [14]. With the addition of multi-epoch data from eight previously unpublished sources, including the four sources in Figure 1, the revised distribution of apparent jet speeds is shown in Figure 2. This Figure incorporates the results of our previously published data and jet speeds for four sources that have been monitored as part of the MOJAVE project [5].

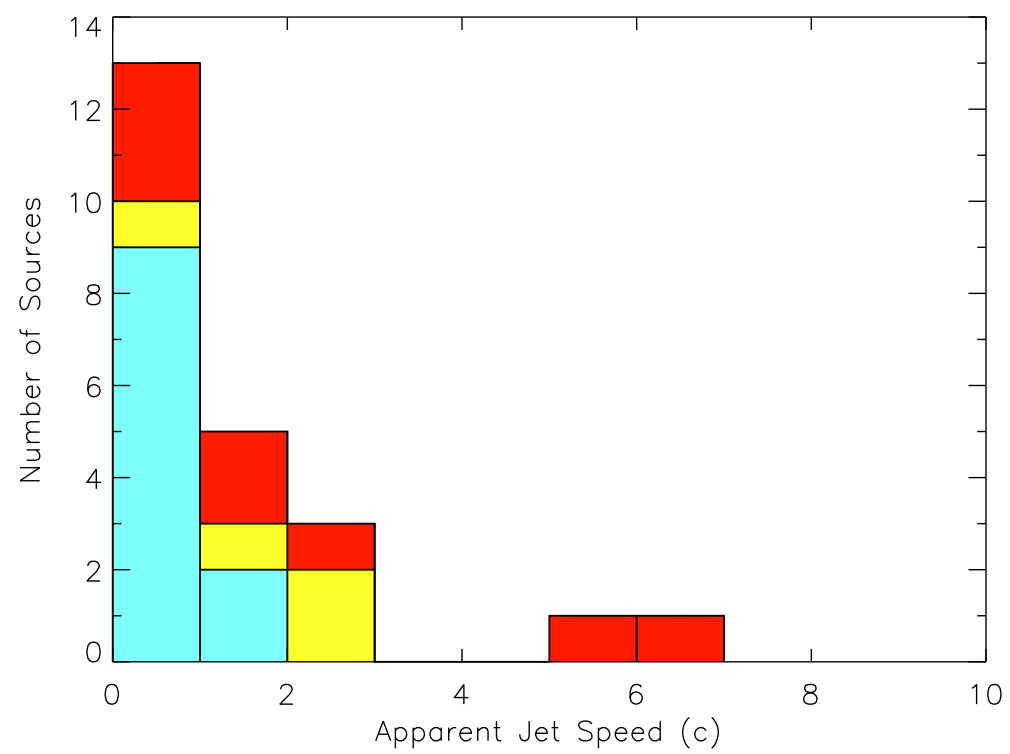

Figure 2. A histogram of the peak jet speeds in TeV blazars. Blue denotes sources from our previously published data, red are from this work, and yellow are from MOJAVE. The highest apparent speed are observed in RBS 0413, $(6.0 \pm 1.2) c$, and RGB J0710+591, (5.8 \pm 1.5$) c$.

With the addition of new data, the tail of the distribution now extends to mildy superluminal apparent speeds for the first time; however, the majority of the TeV HBLs have peak apparent speeds of only about $1 c$. Combining these slow apparent speeds with the high Doppler factors $(\delta)$ implied by the $\mathrm{TeV}$ data to solve for the Lorentz factor $(\Gamma)$ and viewing angle $(\theta)$ results in unreasonbly small viewing angles $\left(\theta<<1^{\circ}\right)$. This would imply tiny jet opening angles, enormous linear sizes, and huge numbers of parent objects, and indicates that the combination of both high Doppler factor and slow apparent speed in the same jet region is unphysical. If more realistic viewing angles of a few degrees are assumed, then the observed apparent speeds imply more modest Lorentz and Doppler factors for the radio jet. The lack of detection of counterjets in the VLBI images for any TeV HBL $[15,23]$ requires that the Doppler factor cannot be arbitrarily low, and values of $\delta$ and $\Gamma$ of a few degrees are most consistent with the combined VLBI data.

\section{Discussion}

A variety of mechanisms have been proposed to try and reconcile the Doppler crisis [24-29]. The most natural explanation is for a range of Doppler factors to coexist in the same jet on parsec scales through jet stratification. One example is a jet that decelerates along its length [24]. In such a jet, the fast inner part sees blueshifted photons from the slower outer part, reducing the high Lorentz factor required in the fast portion. This is a general feature of models with velocity structures; radiative interaction among the different regions allows the SED to be reproduced without the extremely high Lorentz factors and Doppler factors characterizing single-zone models. Another alternative is a transverse velocity structure with a fast central spine and a slower outer sheath. Radiative interaction between the spine and sheath naturally decelerates the spine, producing both radial and transverse velocity structures in the same jet [26]. 
If such spine-sheath jets are present in TeV HBLs, then the outer layer is expected to dominate the radio emission due to its SED shape, even with a lower Doppler factor than the spine [26]. An observational signature of this would be a limb-brightened transverse profile for the jet in VLBI images. There is evidence for limb-brightening close to the core in a number of HBLs, e.g., Mkn 501 [11], 1ES 0502+675 [15], and H 1722+119 [15].

Other possible jet velocity structures have also been proposed, including multiple blobs [30] fast moving "needles" within the main jet [4], "minijets" powered by magnetic reconnection events [31], and turbulent subregions within the jet [29].

\section{Conclusions}

Our on-going VLBA monitoring of the growing number of $\mathrm{TeV}$ gamma-ray emitting HBLs has revealed that the distribution of peak apparent jet speeds in these sources extends to moderate superluminal speeds, $\sim 6 c$, but the majority display subluminal speeds, in contrast with the distribution for other classes of active galactic nuclei [5]. It has recently been proposed that jet kinematics may offer a better classification for blazars than the SED peak frequency [32], with HBLs tending to display quasi-stationary knots arising from recollimation shocks.

A possible physical explanation for this is based on $\mathrm{TeV}$ blazars having intrinsically weak jets that interact with the external medium forming a slow surrounding layer. Radiative interaction between the spine and the sheath decelerates the spine, and eventually disrupts the jet. Such jets are prominent in $\mathrm{TeV}$-selected samples because selection favors rare high-synchrotron peak sources, which are drawn from the low end of the luminosity function where the source density is largest [33].

Acknowledgments: The National Radio Astronomy Observatory is a facility of the National Science Foundation operated under cooperative agreement by Associated Universities, Inc. This research has made use the TeVCat online source catalog (http:/ / tevcat.uchicago.edu). This research has made use of NASA's Astrophysics Data System. This research has made use of the NASA/IPAC Extragalactic Database (NED) which is operated by the Jet Propulsion Laboratory, California Institute of Technology, under contract with the National Aeronautics and Space Administration.

Author Contributions: Glenn Piner and Philip Edwards conceived and designed the observations; Glenn Piner reduced and imaged the data presented in this paper; Philip Edwards prepared the first draft of the paper.

Conflicts of Interest: The authors declare no conflict of interest.

\section{References}

1. Krawczynski, H.; Hughes, S.B.; Horan, D.; Aharonian, F.; Aller, M.F.; Aller, H.; Boltwood, P.; Buckley, J.; Coppi, P.; Fossati, G.; et al. Multiwavelength Observations of Strong Flares from the TeV Blazar 1ES 1959+650. Astrophys. J. 2004, 601, 151-164.

2. Aharonian, F.; Akhperjanian, A.G.; Bazer-Bachi, A.R.; Behera, B.; Beilicke, M.; Benbow, W.; Berge, D.; Bernlöhr, K.; Boisson, C.; Bolz, O.; et al. An Exceptional Very High Energy Gamma-Ray Flare of PKS 2155-304. Astrophys. J. Lett. 2007, 664, L71-L74.

3. Begelman, M.C.; Fabian, A.C.; Rees, M.J. Implications of very rapid TeV variability in blazars. Mon. Not. R. Astron. Soc. 2008, 384, L19-L23.

4. Ghisellini, G.; Tavecchio, F. Rapid variability in TeV blazars: The case of PKS2155-304. Mon. Not. R. Astron. Soc. 2008, 386, L28-L32.

5. Lister, M.L.; Aller, M.F.; Aller, H.D.; Homan, D.C.; Kellermann, K.I.; Kovalev, Y.Y.; Pushkarev, A.B.; Richards, J.L.; Ros, E.; Savolainen, T. MOJAVE: XIII. Parsec-scale AGN Jet Kinematics Analysis Based on 19 years of VLBA Observations at $15 \mathrm{GHz}$. Astron. J. 2016, 152, 12.

6. Ojha, R.; Kadler, M.; Böck, M.; Booth, R.; Dutka, M.S.; Edwards, P.G.; Fey, A.L.; Fuhrmann, L.; Gaume, R.A.; Hase, H.; et al. TANAMI: Tracking active galactic nuclei with austral milliarcsecond interferometry. I. First-epoch 8.4 GHz images. Astron. Astrophys. 2010, 519, A45.

7. Piner, B.G.; Unwin, S.C.; Wehrle, A.E.; Edwards, P.G.; Fey, A.L.; Kingham, K.A. VSOP and Ground-based VLBI Imaging of the TeV Blazar Markarian 421 at Multiple Epochs. Astrophys. J. 1999, 525, 176-190. 
8. Edwards, P.G.; Piner, B.G. The Subluminal Parsec-Scale Jet of Markarian 501. Astrophys. J. Lett. 2002, 579, L67-L70.

9. Giroletti, M.; Giovannini, G.; Feretti, L.; Cotton, W.D.; Edwards, P.G.; Lara, L.; Marscher, A.P.; Mattox, J.R.; Piner, B.G.; Venturi, T. Parsec-Scale Properties of Markarian 501. Astrophys. J. 2004, 600, 127-140.

10. Piner, B.G.; Edwards, P.G. The Parsec-Scale Structure and Jet Motions of the TeV Blazars 1ES 1959+650, PKS 2155-304, and 1ES 2344+514. Astrophys. J. 2004, 600, 115-126.

11. Piner, B.G.; Edwards, P.G. VLBA Polarization Observations of Markarian 421 after a Gamma-Ray High State. Astrophys. J. 2005, 622, 168-177.

12. Piner, B.G.; Pant, N.; Edwards, P.G. The Parsec-Scale Jets of the TeV Blazars H1426+428, 1ES 1959+650, and PKS 2155-304: 2001-2004. Astrophys. J. 2008, 678, 64-77.

13. Piner, B.G.; Pant, N.; Edwards, P.G.; Wiik, K. Significant Limb-Brightening in the Inner Parsec of Markarian 501. Astrophys. J. Lett. 2009, 690, L31-L34.

14. Tiet, V.C.; Piner, B.G.; Edwards, P.G. Increasing the Number of TeV Blazars with Parsec-Scale Kinematics. 2012, arXiv:1205.2399.

15. Piner, B.G.; Edwards, P.G. First-epoch VLBA Imaging of $20 \mathrm{New}$ TeV Blazars. Astrophys. J. 2014, 797, 25.

16. Tavecchio, F. Extragalactic Jets: The High Energy View. In Proceedings of the Tenth Marcel Grossmann Meeting, Rio de Janeiro, Brazil, 20-26 July 2003; Novello, M., Perez Bergliaffa, S., Ruffini, R., Eds.; World Scientific Publishing Co.: Singapore, 2006; p. 512.

17. Henri, G.; Saugé, L. The Bulk Lorentz Factor Crisis of TeV Blazars: Evidence for an Inhomogeneous Pileup Energy Distribution? Astrophys. J. 2006, 640, 185-195.

18. Piner, B.G.; Edwards, P.G. The Parsec-Scale Structure of the Newer TeV Blazars. In Proceedings of the European Physical Journal Web of Conferences, Granada, Spain, 10-14 June 2013; Volume 61, p. 04021.

19. Piner, B.G.; Pant, N.; Edwards, P.G. The Jets of TeV Blazars at Higher Resolution: $43 \mathrm{GHz}$ and Polarimetric VLBA Observations from 2005 to 2009. Astrophys. J. 2010, 723, 1150-1167.

20. Condon, J.J.; Cotton, W.D.; Greisen, E.W.; Yin, Q.F.; Perley, R.A.; Taylor, G.B.; Broderick, J.J. The NRAO VLA Sky Survey. Astron. J. 1998, 115, 1693-1716.

21. Aharonian, F.; Akhperjanian, A.G.; Bazer-Bachi, A.R.; Beilicke, M.; Benbow, W.; Berge, D.; Bernlöhr, K.; Boisson, C.; Bolz, O.; Borrel, V.; et al. A low level of extragalactic background light as revealed by $\gamma$-rays from blazars. Nature 2006, 440, 1018-1021.

22. Stecker, F.W.; Scully, S.T.; Malkan, M.A. An Empirical Determination of the Intergalactic Background Light from UV to FIR Wavelengths Using FIR Deep Galaxy Surveys and the Gamma-Ray Opacity of the Universe. Astrophys. J. 2016, 827, 6.

23. Giroletti, M.; Giovannini, G.; Cotton, W.D.; Taylor, G.B.; Pérez-Torres, M.A.; Chiaberge, M.; Edwards, P.G. The jet of Markarian 501 from millions of Schwarzschild radii down to a few hundreds. Astron. Astroph. 2008, 488, 905-914.

24. Georganopoulos, M.; Kazanas, D. Decelerating Flows in TeV Blazars: A Resolution to the BL Lacertae-FR I Unification Problem. Astrophys. J. Lett. 2003, 594, L27-L30.

25. Gopal-Krishna; Dhurde, S.; Wiita, P.J. Do the Mildly Superluminal VLBI Knots Exclude Ultrarelativistic Blazar Jets? Astrophys. J. Lett. 2004, 615, L81-L84.

26. Ghisellini, G.; Tavecchio, F.; Chiaberge, M. Structured jets in TeV BL Lac objects and radiogalaxies. Implications for the observed properties. Astron. Astrophys. 2005, 432, 401-410.

27. Gopal-Krishna; Wiita, P.J.; Dhurde, S. Bulk motion of ultrarelativistic conical blazar jets. Mon. Not. R. Astron. Soc. 2006, 369, 1287-1292.

28. Gopal-Krishna; Dhurde, S.; Sircar, P.; Wiita, P.J. Influence of the jet opening angle on the derived kinematical parameters of blazar jets having uniform and stratified bulk motion. Mon. Not. R. Astron. Soc. 2007, $377,446-452$.

29. Marscher, A.P. Turbulent, Extreme Multi-zone Model for Simulating Flux and Polarization Variability in Blazars. Astrophys. J. 2014, 780, 87.

30. Tavecchio, F.; Becerra-Gonzalez, J.; Ghisellini, G.; Stamerra, A.; Bonnoli, G.; Foschini, L.; Maraschi, L. On the origin of the $\gamma$-ray emission from the flaring blazar PKS 1222+216. Astron. Astrophys. 2011, 534, A86.

31. Giannios, D. Reconnection-driven plasmoids in blazars: Fast flares on a slow envelope. Mon. Not. R. Astron. Soc. 2013, 431, 355-363. 
32. Hervet, O.; Boisson, C.; Sol, H. An innovative blazar classification based on radio jet kinematics. Astron. Astrophys. 2016, 592, A22.

33. Giommi, P.; Padovani, P.; Polenta, G.; Turriziani, S.; D’Elia, V.; Piranomonte, S. A simplified view of blazars: Clearing the fog around long-standing selection effects. Mon. Not. R. Astron. Soc. 2012, 420, 2899-2911.

(C) 2016 by the authors; licensee MDPI, Basel, Switzerland. This article is an open access article distributed under the terms and conditions of the Creative Commons Attribution (CC-BY) license (http:/ / creativecommons.org/licenses/by/4.0/). 